\title{
Analysis of Low Power Multi-core Embedded Management for Energy Harvesting
}

\author{
M.Valan Rajkumar ${ }^{1}$, T.Indumathi ${ }^{2}$ \\ ${ }^{1,2}$ (Department of Electrical and Electrical Engineering, Gnanamani College of Technology, Namakkal, India)
}

\begin{abstract}
This paper presents an Intelligent Power management system which primarily focuses on power harvesting and low power consumption management and controlling of multi-core embedded system. Now, such multi-core embedded systems range from portable devices such as large stationary installations, factory controllers, and largely complex systems like hybrid vehicles, MRI, and avionics. Its Complexity varies from low with single microcontroller chip to very high with multiple units, peripherals and networks mounted inside a large chassis or enclosure. The multi-core embedded controller implements the Maximum Power Point Tracking (MPPT) algorithm based fuzzy logic control for extracting the maximum power from the PV cell. Further it is improved as user interaction by the provision of GSM transceiver, Wireless technologies and Sensing etc...
\end{abstract}

Keywords: DC-DC converter, Fuzzy control, Maximum Power Point Tracking (MPPT) algorithm, Photovoltaic (PV) cells, Power management.

\section{Introduction}

Many Prior works have focused on the problem of fuzzy logic control for real- time embedded systems. Here, we only discuss the few works are relevant to our goals. An early work FLC has been introduced in many researches as [1-2] addressed the problem of MPPT based Fuzzy logic controller using line-commutated inverter for three-phase grid-connected photovoltaic systems. In [2], algorithms were proposed to minimize the energy management of a domestic photovoltaic panel using the algorithm of multi-criteria fuzzy algorithm. In [3-4] used to improve the maximum power for photovoltaic solar energy systems using fuzzy logic controller. At the above fuzzy controller are used to improve the pv systems power. Here fuzzy cognitive networks (FCN) is used to maximize the power point tracking method for PV systems [5]. In [6], PV applications variable step-size is implemented by using FPGA implementation using $\mathrm{P} \& \mathrm{O}$ algorithm of Maximum Power Point Tracking. In this paper [6], Multi-fuzzy logic controllers determine and analysis the efficiency of a photovoltaic system in its operating point. The fuzzy controller neural-network-based maximum-power-point tracking is coupled-inductor interleaved-boost converter-supplied PV system [7-10].

A typical battery-powered portable device not stands for more than many days in active mode. Few days only battery power is used and frequently battery change is not desirable for many applications such as remote sensing and monitoring system, unmanned vehicle, etc. To attain a continuous supply we can use photovoltaic supply instead of battery power. The system can efficiently control the power delivered from the sources to the embedded controller. Energy harvesting are mostly used for the source of rechargeable and exchangeable for batteries, self-sustainable energy supply is favor of emerging embedded systems of pervasive and efficient computing. PV cell is used as the primary source of energy harvesting. A battery is used to store the power generated by the energy sources. Here the saved battery power from the solar system is utilized supplanting of portable power supply [11-14]. The user is also given the control to switch on and off the sources, thus acting as the governing unit for the entire system.

In this paper, we propose an ARM CORTEX-M controller is used for all control purposes which provides us with many intuitive solutions. This implements the popularly used MPPT algorithms Perturb and Observe (P\&O), Incremental Conductance (INC) algorithm [15-19] on the PV array which powers the controller. Adaptive Neuro Fuzzy Inference System (ANFIS) based controller switching scheme is used to improve the power quality thus to reduce the Total Harmonic Distortion (THD) [20-26]. The power variation and output voltage variation are chosen as inputs of the proposed FLC, which simplifies the calculation. Compared with the conventional perturb and observe (P\&O) method, the proposed FLC-based MPPT can simultaneously improve the dynamic and steady state performance of the PV system. The other drawback of Solar PV systems is the variation in output voltage with variations in solar radiation and temperature. Several techniques and algorithms have been proposed in this area to overcome the drawbacks of the Solar PV system. PV modules have unique power Vs voltage (P-V) characteristics. From the P-V characteristics, PV systems must be operated at a maximum power point (MPP) of specific current and voltage values so as to increase the PV efficiency. For any PV system, the output power can be increased by tracking the MPP of the PV module by using a controller connected to a chopper. One of such techniques is the use of Fuzzy Logic controllers in 
Maximum Power Point Tracking of the Solar PV systems. The Maximum power point changes with the variation in the solar radiation and temperature, so FLC is used to force the PV system to operate at the maximum power point. A highly robust Fuzzy Logic based controller is designed in MATLAB/ Simulink environment. The FLC is used in Solar PV system along with a DC-DC chopper to increase the efficiency of the Solar PV system. In the Fuzzy Logic Controller the modifications are done in Rule base and membership functions according to the variations in solar radiation and temperature. The output of the FLC is the change in the duty cycle of the DC-DC chopper. To further improves the performance of the proposed method, an asymmetrical membership function (MF) concept is also proposed. Two design procedures are proposed to determine the universe of discourse (UOD) of the input MF. Many MPPT methods have been developed and implemented in previous studies, including perturb and observe ( $\mathrm{P} \& \mathrm{O})$, incremental conductance (INC), fractional open-circuit voltage, fractional short-circuit current, line approximation, ripple correlation control (RCC) and fuzzy logic control (FLC) approaches. These techniques have high tracking accuracy under steady weather conditions, but still exhibit some trade-offs between tracking speed and tracking accuracy when insulation changes.

\subsection{Modeling of Photovoltaic System}

\section{Design of Photovoltic System}

Due to energy crisis and environmental issues such as pollution and global warming effect, photovoltaic (PV) systems are becoming a very attractive solution. Unfortunately the actual energy conversion efficiency of PV module is rather low. So to overcome this problem and to get the maximum possible efficiency, the design of all the elements of the PV system has to be optimized shown in Figure 1. In this paper, we focus on the problem of power and thermal management of real time embedded system with periodic tasks, powered by solar energy. To cope with the unstable nature of the solar energy source, a super capacitor is used to buffer solar energy controlled by photovoltaic cells.



Figure 1. Equivalent Circuit diagram of PV cell

\subsection{DC-TO-DC Converter Topology}

A DC-to-DC converter is an electronic circuit which converts a source of direct current (DC) from one voltage level to another. It is a class of power converter. DC to DC converters are important in portable electronic devices such as cellular phones and laptop computers, which are supplied with power from batteries primarily. Such electronic devices often contain several sub-circuits, each with its own voltage level requirement different from that supplied by the battery or an external supply (sometimes higher or lower than the supply voltage). Additionally, the battery voltage declines as its stored power is drained. Switched DC to DC converters offer a method to increase voltage from a partially lowered battery voltage thereby saving space instead of using multiple batteries to accomplish the same thing. Most DC to DC converters also regulate the output voltage. Some exceptions include high-efficiency LED power sources, which are a kind of DC to DC converter that regulates the current through the LEDs, and simple charge pumps which double or triple the output voltage.

Electronic switch-mode DC to DC converters convert one DC voltage level to another, by storing the input energy temporarily and then releasing that energy to the output at a different voltage. The storage may be in either magnetic field storage components (inductors, transformers) or electric field storage components (capacitors). This conversion method is more power efficient (often $75 \%$ to $98 \%$ ) than linear voltage regulation (which dissipates unwanted power as heat). This efficiency is beneficial to increasing the running time of battery operated devices. The efficiency has increased since the late 1980s due to the use of power FETs, which are able to switch at high frequency more efficiently than power bipolar transistors, which incur more switching losses and require a more complicated drive circuit. Another important innovation in DC-DC converters is the use of synchronous rectification replacing the flywheel diode with a power FET with low "on resistance", thereby reducing switching losses. Before the wide availability of power semiconductors, low power DC to DC converters of this family consisted of an electro-mechanical vibrator followed by a voltage step-up transformer and a vacuum tube or semiconductor rectifier or synchronous rectifier contacts on the vibrator. 


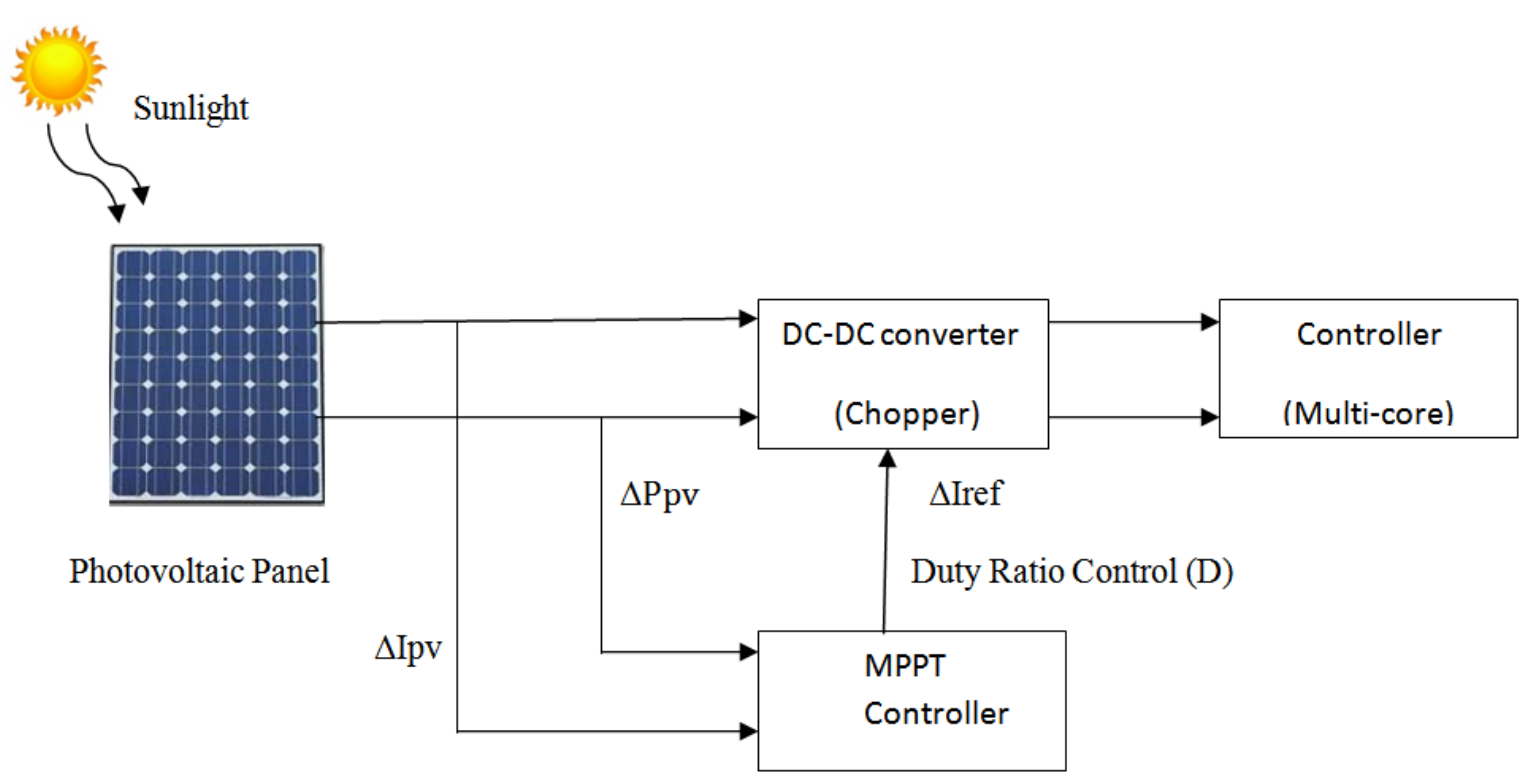

Figure 2. Solar Power with Maximum Power Tracking System

In Figure 2, to verify the correctness of the proposed FLC-based MPPT controller, a $300 \mathrm{~W}$ prototyping circuit is implemented from which experiments are carried out accordingly. The proposed algorithm is validated using an AMETEK Solar Array Simulator TerraSAS DCS80-15 in SAS mode. The parameters of the utilized PV panel are listed and the specification of the utilized power converter is also listed. In order to validate the effectiveness of the proposed MPPT controller, experiments will be carried out on the following systems: Two P\&O MPPT methods with different perturbation step settings, one proposed symmetrical FLCbased MPPT method and two proposed asymmetrical FLC-based MPPT methods with different settings. All experiments are conducted using the same power circuit to ensure the fairness. It shows the parameters used in each MPPT algorithm. Using a $40 \mathrm{MHz}$ oscillator, the required execution times of the P\&O MPPT, the symmetrical FLC-based MPPT and the asymmetrical FLC-based MPPT are $1.5 \mu \mathrm{s}, 120 \mu \mathrm{s}$ and $120 \mu \mathrm{s}$, respectively. It shows the starting waveform of these five methods for $1000 \mathrm{~W} / \mathrm{m} 2$ solar irradiance and $25^{\circ} \mathrm{C}$ PV panel temperature. From the transient times for the fixed step P\&O method are $250 \mathrm{~ms}$ (for 5\% fixed step) and $3.13 \mathrm{~s}$ (for $0.5 \%$ fixed step), respectively. Additionally, the transient time for the proposed symmetrical FLC-based MPPT controller, the proposed asymmetrical FLC-based MPPT controller are $1.59 \mathrm{~s}, 968 \mathrm{~ms}$ and $913 \mathrm{~ms}$, respectively. Observing From, the P\&O method with large step exhibits good dynamic performance but larger steady state oscillations; therefore, the MPPT accuracy is rather low. On the other hand, using a small step size can improve the tracking accuracy at the cost of slower dynamic performance. The proposed FLC-based MPPT methods solves this dilemma as evident from Figure shown. It can be seen from Figure that although the transient time of the proposed method is slightly higher than the P\&O method using large step size, the oscillation around the MPP for the proposed method is much smaller than that obtained from the P\&O method using large step size.

\section{Fuzzy Logic Control Based MPPT Algorithm}

Conversion Monitoring Circuitry of MPPT measures and modulates the output from the EH Module and direct the energy flow among the EH, ES and RTES modules. Capable of performing automatic maximum power point tracking (MPPT) is to yield the maximum output power.

The principle of this controller is to provoke perturbation by acting (decrease or increase) on the PWM duty cycle and observing the effect on the output PV power. If the instant power $\mathrm{P}(\mathrm{k})$ is greater than the previous computed power $\mathrm{P}(\mathrm{k}-1)$, then the direction of perturbation is maintained otherwise it is reversed. Power- Voltage characteristics of PV module can be detailed as follows: when $\mathrm{dp} / \mathrm{dv}>0$, the voltage is increased, this is done through $\mathrm{D}(\mathrm{k})=\mathrm{D}(\mathrm{k}-1)+\mathrm{C},(\mathrm{C}$ - incrementation step), when $\mathrm{dp} / \mathrm{dv}>0$, the voltage is decreased, this is done through $\mathrm{D}(\mathrm{k})=\mathrm{D}(\mathrm{k}-1)-\mathrm{C}$. To simulate this $\mathrm{P} \& \mathrm{O}$ algorithm, a boost chopper, as a DC-DC converter which is described by the equations (1), (2) \& (3):

$$
\begin{gathered}
i l=i-C_{l} \frac{d v}{d t} \\
i b=(1-D) i t-C 2 \frac{d v b}{d t} \\
\mathrm{~V}=(1-D) \mathrm{Vb}+\mathrm{L} \frac{d i f}{d t}
\end{gathered}
$$


This study made a through investigation on common fuzzy input variables for fuzzy logic based MPPT algorithms by reviewing the characteristics of PV cells and past literature on fuzzy MPPT controller designs shown in Figure 3. Advantages and disadvantages of various designs were reviewed and provided with detailed discussions on issues encountered during the design process. Finally, the designs were verified using computer simulation.

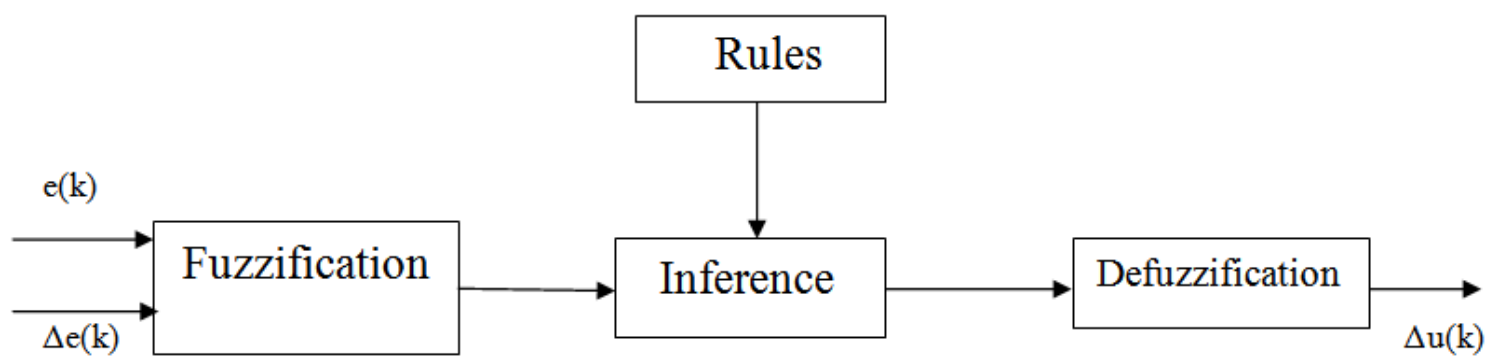

Figure 3. Diagram of Fuzzy Controller

\subsection{Algorithm (i): P-V Slope and Variation of Slope as the Inputs}

Algorithm (i) of the fuzzy logic MPPT system used the slope of the PV cell's Power-Voltage (P-V) curve $(S(k))$ and variation of slope $(\Delta S(k))$ as the fuzzy input variables. In Figure 4, these variables were defined using the following equations (4) \& (5):

$$
s(k)=\frac{\Delta P p v}{\Delta V p v}=\frac{\lfloor p v(k) \cdot V p v(k)-I p v(k-1) \cdot V p v(k-1)]}{[V p v(k)-V p v(k-1)]}
$$

$$
\Delta S(k)=S(k)-S(k-1)
$$

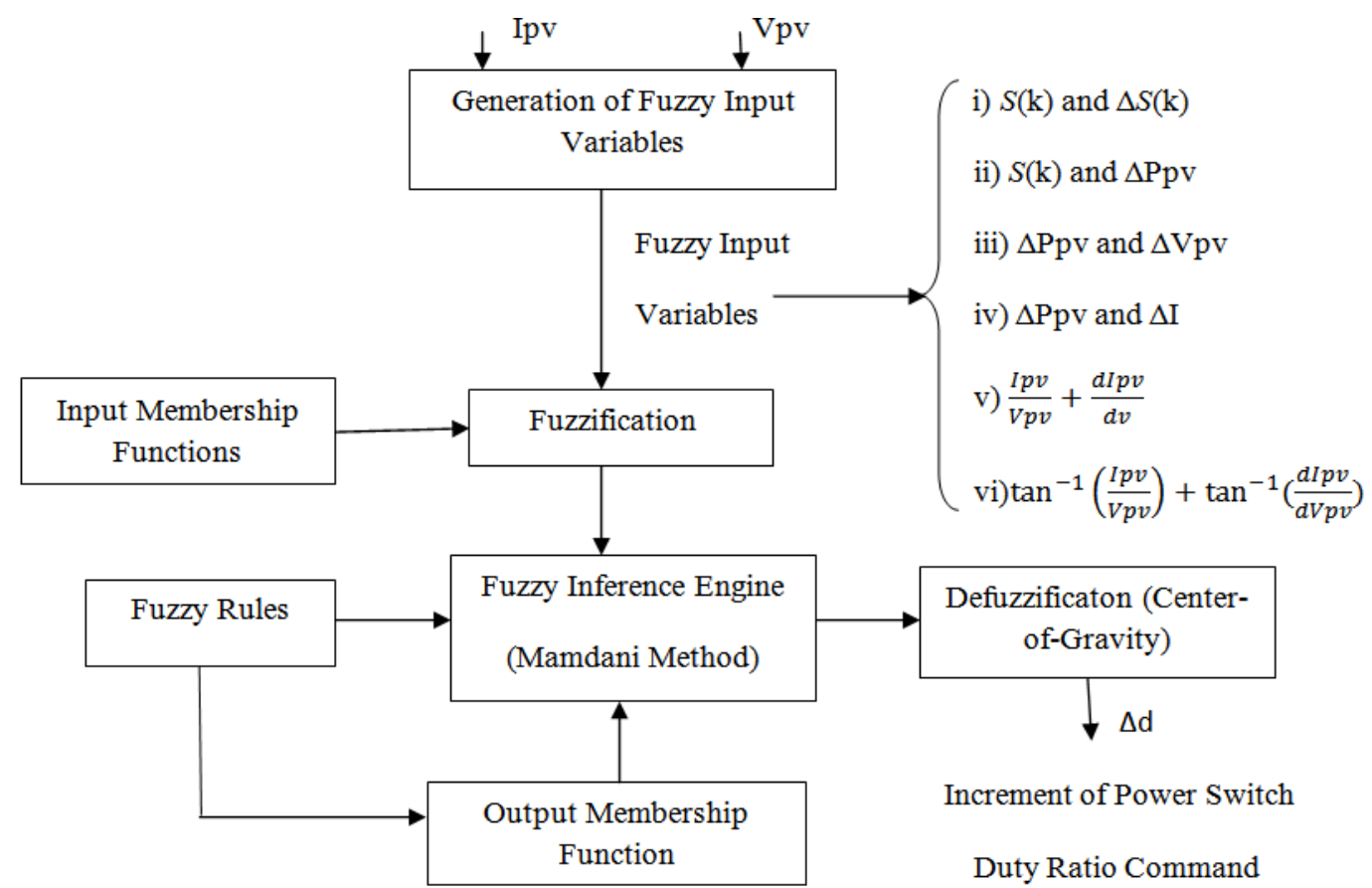

Figure 4. Flow diagram of Fuzzy Controller

The database for fuzzy rules designed according to the fuzzy input variables. A five-term fuzzy set, positive big (PB), positive small (PS), zero (ZE), negative small (NS), and negative big (NB), is defined to describe each linguistic variable. Output from the fuzzy controller (duty ratio command of the buck-boost converter) would change the output voltage and current of the PV cell. Once PV cell outputs change, it would affect the values of the next round of fuzzy input variables. The controller would then re-adjust the output commands accordingly. According to fuzzy logic, the selection of the domain of the inputs and outputs 
(universe of discourse) will also directly affect the results, so careful designs must be implemented. The general guidelines for determining the membership functions are: (1) defining the boundaries of the PB and NB regions first based the characteristics of the input variables; (2) the range of ZE is then determined based on the predetermined MPPT goal (efficiency criteria); (3) the boundaries of PM and NM are then determined following the selection of the boundaries of PB, NB, and ZE. Design iterations are usually required to reach a satisfactory result.

\subsection{Algorithm (ii): P-V Slope and Variation of Power as the Inputs}

Algorithm (ii) of the fuzzy logic MPPT system used the slope $S(k)$ and variation of power $(\triangle P p v)$ as the fuzzy input variables. The fuzzy rules database was divided into 4 regions according to the characteristics of the P-V curve of the PV cell. Detailed analysis and discussions are provided below.

Region1. The main determinant in this region is a negative slope, with the operating point located on the right side of the MPP. Hence, the system is able to conclude that duty ratio needs to be increased to track the MPP. Variation to power output would be used to acquire the magnitude of increase for the duty ratio.

Region2. The slope is ZE in this region. Duty ratio would thus remain unchanged.

Region3. The main determinant in this region is a positive slope, with the operating point located on the left side of the MPP. Hence, the system is able to conclude that duty ratio needs to be decreased to track the MPP. Variation to power output would be used to acquire the magnitude of decrease for the duty ratio.

Region4. This region mainly determines the responses implemented when changes to the power output has been determined to be ZE. When variation to power is ZE, the operating point would be very close to the MPP. At this time, $S(k)$ could be used to improve the precision of the operating point. The use of $S(k)$ specifically targets low irradiation levels where the P-V curve has very low slope and that the system may be unable to accurately perform MPPT. Hence, the slope was used to improve the accuracy and precision of the system algorithm. The designed increment or decrease of each duty ratio would be small in order to prevent adding or removing too much duty ratio in a single step that could give rise to fluctuations of the operating point.

\section{Design And Simulation Analysis}

In one simple inverter circuit, DC power is connected to a transformer through the centre tap of the primary winding. A switch is rapidly switched back and forth to allow current to flow back to the DC source following two alternate paths through one end of the primary winding and then the other. The alternation of the direction of current in the primary winding of the transformer produces alternating current (AC) in the secondary circuit.

The electromechanical version of the switching device includes two stationary contacts and a spring supported moving contact. The spring holds the movable contact against one of the stationary contacts and an electromagnet pulls the movable contact to the opposite stationary contact. The current in the electromagnet is interrupted by the action of the switch so that the switch continually switches rapidly back and forth. This type of electromechanical inverter switch, called a vibrator or buzzer, was once used in vacuum tube automobile radios. A similar mechanism has been used in door bells, buzzers and tattoo guns. As they became available with adequate power ratings, transistors and various other types of semiconductor switches have been incorporated into inverter circuit designs.

\subsection{Advanced Designs of Multiple Inverter}

There are many different power circuit topologies and control strategies used in inverter designs. Different design approaches address various issues that may be more or less important depending on the way that the inverter is intended to be used.

The issue of waveform quality can be addressed in many ways. Capacitors and inductors can be used to filter the waveform. If the design includes a transformer, filtering can be applied to the primary or the secondary side of the transformer or to both sides. Low-pass filters are applied to allow the fundamental component of the waveform to pass to the output while limiting the passage of the harmonic components. If the inverter is designed to provide power at a fixed frequency, a resonant filter can be used. For an adjustable frequency inverter, the filter must be tuned to a frequency that is above the maximum fundamental frequency.

Since most loads contain inductance, feedback rectifiers or anti-parallel diodes are often connected across each semiconductor switch to provide a path for the peak inductive load current when the switch is turned off. The anti-parallel diodes are somewhat similar to the freewheeling diodes used in AC/DC converter circuits.

Fourier analysis reveals that a waveform, like a square wave, that is anti-symmetrical about the 180 degree point contains only odd harmonics, the 3rd, 5th, 7th etc. Waveforms that have steps of certain widths and heights eliminate or "cancel" additional harmonics. For example, by inserting a zero-voltage step between the positive and negative sections of the square-wave, all of the harmonics that are divisible by three can be 
eliminated. That leaves only the 5th, 7th, 11th, 13th etc. The required width of the steps is one third of the period for each of the positive and negative steps and one sixth of the period for each of the zero-voltage steps.

Changing the square wave as described above is an example of pulse-width modulation (PWM). Modulating, or regulating the width of a square-wave pulse is often used as a method of regulating or adjusting an inverter's output voltage. When voltage control is not required, a fixed pulse width can be selected to reduce or eliminate selected harmonics. Harmonic elimination techniques are generally applied to the lowest harmonics because filtering is more effective at high frequencies than at low frequencies. Multiple pulse-width or carrier based PWM control schemes produce waveforms that are composed of many narrow pulses. The frequency represented by the number of narrow pulses per second is called the switching frequency or carrier frequency. These control schemes are often used in variable-frequency motor control inverters because they allow a wide range of output voltage and frequency adjustment while also improving the quality of the waveform.

Multilevel inverters provide another approach to harmonic cancellation. Multilevel inverters provide an output waveform that exhibits multiple steps at several voltage levels. For example, it is possible to produce a more sinusoidal wave by having split-rail direct current inputs at two voltages, or positive and negative inputs with a central ground. By connecting the inverter output terminals in sequence between the positive rail and ground, the positive rail and the negative rail, the ground rail and the negative rail, then both to the ground rail, a stepped waveform is generated at the inverter output. This is an example of a three level inverter: the two voltages and ground.

\subsection{MATLAB Simulation}

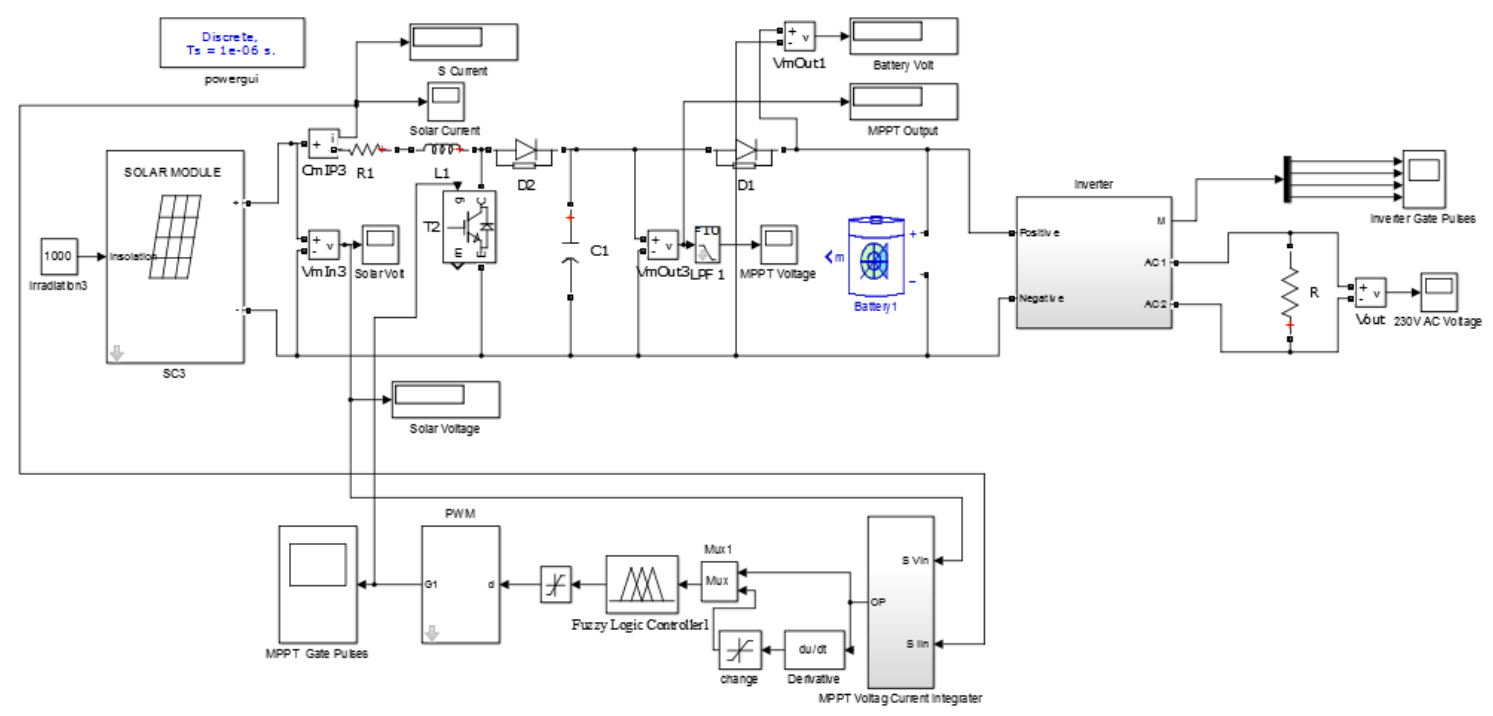

Figure 5. Circuit Diagram for MPPT using Fuzzy Logic

\subsection{Embedded System Programming}

ARM Holdings neither manufactures nor sells CPU devices based on its own designs, but rather licenses the processor architecture to interested parties. ARM offers a variety of licensing terms, varying in cost and deliverables. To all licensees, ARM provides an integratable hardware description of the ARM core, as well as complete software development toolset and the right to sell manufactured silicon containing the ARM CPU. Some of the most important options for the Cortex-M cores are SysTick timer is 24-bit system timer that extends the functionality of both the processor and the Nested Vectored Interrupt Controller (NVIC). When present, it also provides an additional configurable priority SysTick interrupt. Though the SysTick timer is optional, it's rare to see a Cortex-M microcontroller without it. Bit-banding is maps a complete word of memory onto a single bit in the bit-band region. For example, writing to an alias word will set or clear the corresponding bit in the bit band region. This allows every individual bit in the bit-banding region to be directly accessible from a word-aligned address, and individual bits to be toggled from $\mathrm{C} / \mathrm{C}++$ without performing a read-modifywrite sequence of instructions. Memory Protection Unit (MPU) is Provides support for protecting regions of memory through enforcing privilege and access rules. It supports up to eight different regions, each of which can be split into a further eight equal-size sub-regions.

In Figure $6 \& 7$ shows, The Cortex-M7 implements the ARMv7E-M micro architecture. The architectures are binary instruction upward compatible from ARMv6-M to ARMv7E-M. Binary instructions available for execution on the Cortex-M7.The Cortex-M7 have entire base Thumb instructions (CBZ, CBNZ, IT) and a minor subset of Thumb-2 instructions (BL, DMB, DSB, ISB, MRS, MSR). The Cortex-M3 adds 3 
Thumb instructions, all Thumb-2 instructions, hardware divide, and saturation arithmetic instructions. The Cortex-M4 adds DSP instructions and an optional single-precision floating-point unit (VFPv4-SP). The CortexM7 adds an optional double-precision Floating Point Unit (VFPv5). The single-precision (SP) FPU instructions are valid in the Cortex-M4/M7 only when the SP FPU option exists in the silicon. The double-precision (DP) FPU instructions are valid in the Cortex-M7 only when the DP FPU option exists in the silicon. Only Thumb and Thumb-2 instruction sets are supported in Cortex-M architectures, but the legacy 32-bit ARM instruction set isn't supported. Cortex-M7 (F) supported for the instruction set of Thumb, Thumb-2, Saturated Math, DSP extensions, FPU (SP \& DP) (M7F). The Cortex-M7 is a high-performance core with almost double the power efficiency of the older Cortex-M4. It features a 6-stage superscalar pipeline with branch prediction/speculation and an optional floating-point unit capable of single-precision and optionally double-precision operations. The instruction and data buses have been enlarged to 64-bit wide over the previous 32-bit buses. If a core contains an FPU, it is known as a Cortex-M7 (F), otherwise it is a Cortex-M7. 32-bit hardware multiply with 32-bit or 64-bit result, signed or unsigned, add or subtract after the multiply and 32-bit hardware divide (2-12 cycles). Also has the key feature of saturation arithmetic support. DSP extension is single cycle 16/32-bit MAC, single cycle dual 16-bit MAC, 8/16-bit SIMD arithmetic. Interrupts plus NMI has 1 to 240 interrupts and 12 cycles interrupt latency. It includes integrated sleep modes.

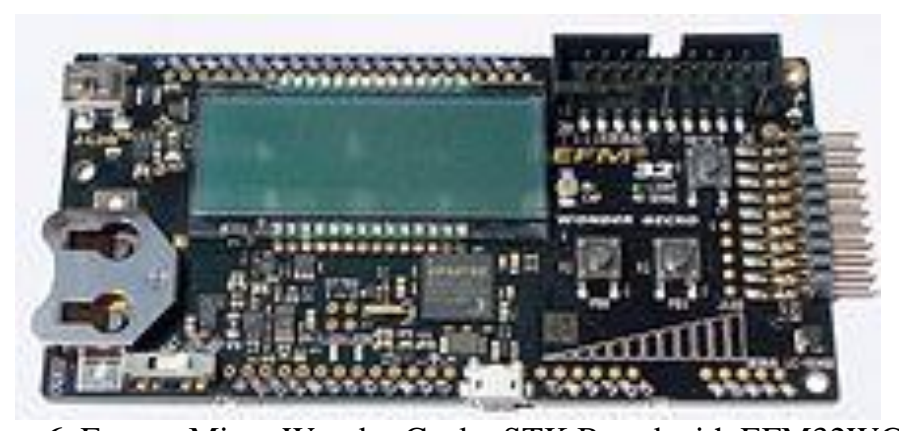

Figure 6. Energy Micro Wonder Gecko STK Board with EFM32WG990

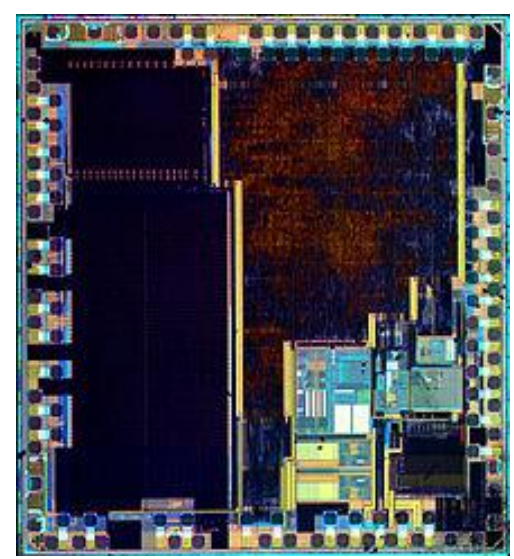

Figure 7. STM32F100C4T6B ARM Cortex-M3 microcontroller

Silicon options as Optional Floating-Point Unit (FPU) in (single precision) or (single and doubleprecision), both IEEE-754-2008 compliant. It is called the FPv5 extension. Optional CPU cache has 0 to 64 KB instruction-cache, 0 to $64 \mathrm{~KB}$ data-cache, each with optional ECC. Optional Tightly-Coupled Memory (TCM) contains 0 to $16 \mathrm{MB}$ instruction-TCM, 0 to $16 \mathrm{MB}$ data-TCM, each with optional ECC. Optional Memory Protection Unit (MPU) includes 8 or 16 regions. Optional Embedded Trace Macrocell (ETM) has instructiononly or instruction and data. Optional Retention Mode (with ARM Power Management Kit) for Sleep Modes.The following microcontrollers are based on the Cortex-M7 core such as Atmel SAME70, SAMS70, SAMV70, Freescale/NXP Kinetis KV5x, STMicroelectronics STM32 F7.

\section{Experimental Result}

One simple solar panel that has standard value of isolation and temperature has been included in the simulation circuit. From all the cases, the best controller for MPPT is incremental conductance controller. This controller gives a better output value for buck, boost converter. Hence this controller will give different kind of curves for the entire converter. In simulation Buck converter show the best performance the controller work at the best condition using buck controller. 


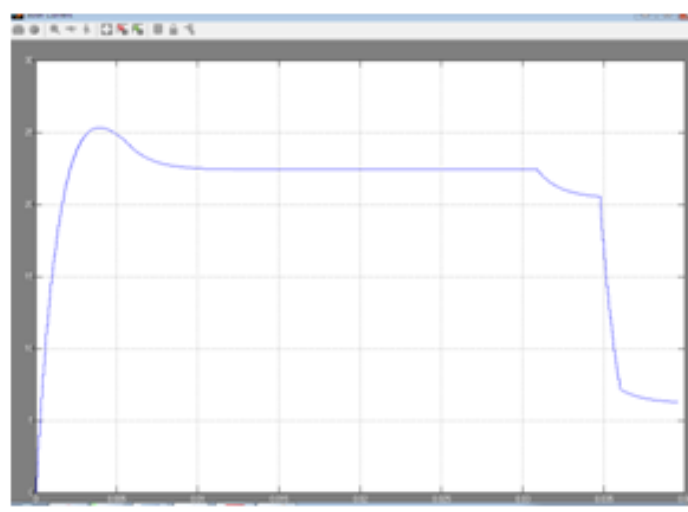

Figure 8. Output of Solar Current

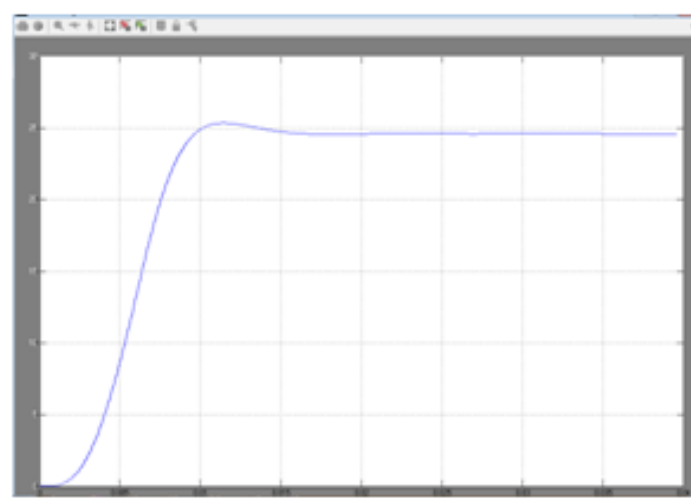

Figure 10. Output of MPPT Voltage

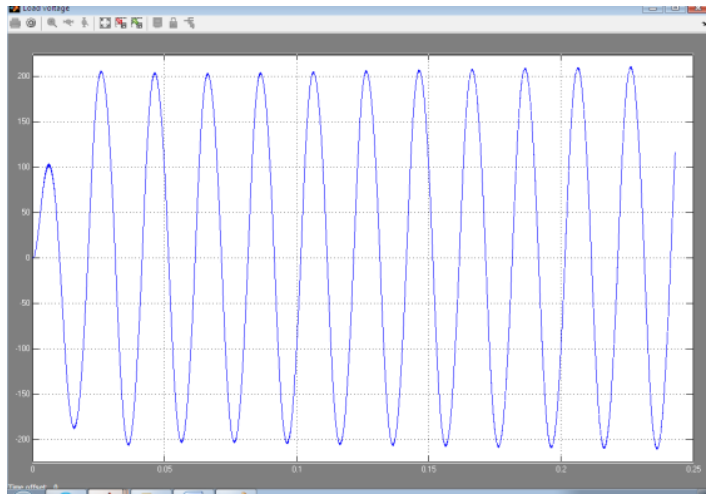

Figure 12. Inverter output voltage

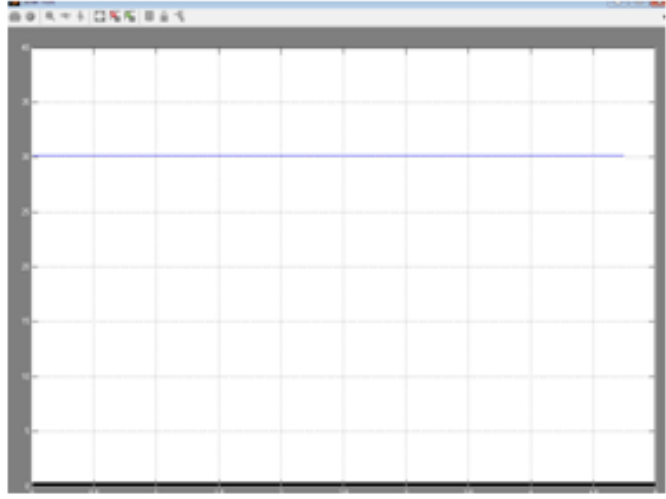

Figure 9. Output of Solar Voltage

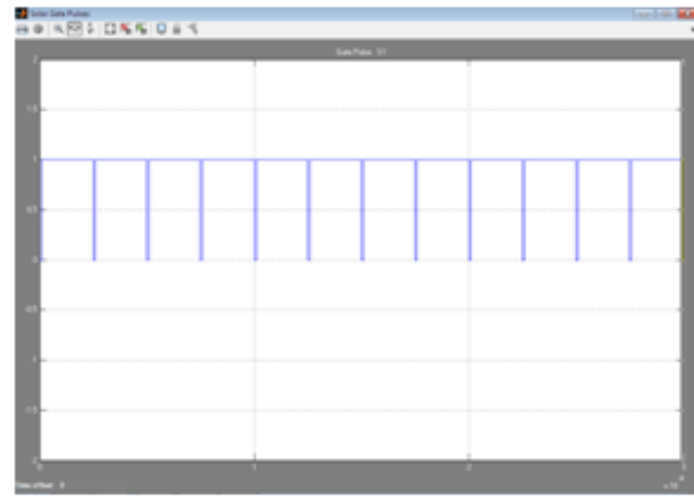

Figure 11. MPPT Gate Pulse

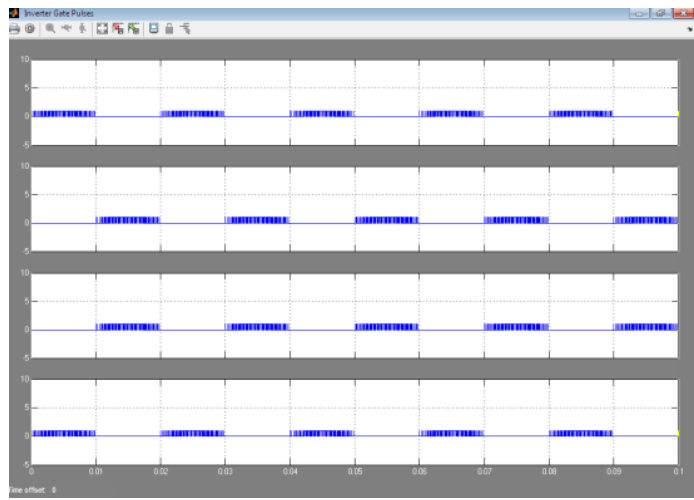

Figure 13. Inverter gate pulse

\section{Conclusion}

In this paper, we proposed a novel framework for runtime energy and workload management of MPPT based on Fuzzy logic control, for real-time multi-core embedded systems with solar energy harvesting. Compared with the best known previous work, our approach is promising for energy-harvesting-based multicore embedded systems. The designs of six fuzzy MPPT algorithms using different input variables. Detailed considerations, determinations of the fuzzy rules associated with the different fuzzy input variables, as well as the advantages and disadvantages of the algorithms have also been summarized in the paper. Algorithms are used the sum of the angles of the arctangent of the conductance and the arctangent of the incremental conductance as the input variable, was considered the most promising MPPT algorithm according to our considerations as the MPP conditions would have a well-defined range for both the sum $\left(180^{\circ}\right)$ and the range of the sum $\left(90 \sim 270^{\circ}\right)$. When compared to other algorithms, it made it easier to determine the range of the universe of discourse, fuzzy rules, and the associated membership functions for the MPPT algorithm. Computer simulation also confirmed that Algorithms are provided better MPPT performance. Additionally, the MPPT function could also be formulated as a form of feedback control using $180 \mathrm{o}$ as the reference input and be applied in multipurpose controller designs such as by incorporating of voltage regulation purposes. 


\section{References}

[1] H.Patel and V.Agarwal, "Maximum power point tracking scheme for PV systems operating under partially shaded conditions," IEEE Trans.Ind. Electron.,vol.55,no.4,pp.302 to 310,Apr.2008.

[2] B.Subudhi and R. Pradhan,"A comparative study on maximum powerpoint tracking techniques for photovoltaic power systems,"IEEE Trans. Sustain.Energy,vol.4,no.1,pp.89-98,Jan.2013.

[3] M.Valan Rajkumar, P.S.Manoharan, Modeling and Simulation of Three-phase DCMLI using SVPWM for Photovoltaic System, Springer Lecture Notes in Electrical Engineering, under the volume titled "Power Electronics \& Renewable Energy Systems", Volume 326, Chapter No 5, January 2015, Pages 39-45.

[4] M.Valan Rajkumar, P.S.Manoharan, Harmonic Reduction of Fuzzy PI Controller based Three-Phase Seven-level DCMLI with SVPWM for Grid Connected Photovoltaic System, Journal International Review on Modeling and Simulations, Volume 6, No 3, June 2013, Pages 684-692.

[5] M.A.Elgendy, B.Zahawi, and D.J.Atkinson, "Assessment of perturband observe MPPT algorithm implementation techniques for PV pumpingapplications,” IEEE Trans. Sustain. Energy, vol. 3, no.1, pp.21-31,Jan.2012.

[6] M.A.Elgendy,B.Zahawi, and D. J. Atkinson, "Operating characteristicsof the P\&O algorithm at high perturbation frequencies for standalone PVsystems,” IEEE Trans. Energy Convers., vol. 30, no. 1, pp. 189-198, Jun.2015.

[7] M.Valan Rajkumar, P.S.Manoharan, FPGA Based Multilevel Cascaded Inverters with SVPWM Algorithm for Photovoltaic system, Elsevier Journal Solar Energy, Volume 87, Issue 1, January 2013, Pages 229-245.

[8] R. Kotti andW. Shireen, "EfficientMPPT control for PV systems adaptive to fast changing irradiation and partial shading conditions," Solar Energy, vol.114, pp.397-407, Mar. 2015.

[9] K. Ishaque, Z. Salam, M. Amjad, and S. Mekhilef, “An improved particle swarm optimization (PSO)-based MPPT for PV with reduced steady-state oscillation,” IEEE Trans. Power Electron., vol. 27, no. 8,pp. 3627 to 3638, Aug. 2012.

[10] P.Thirumurugan, P.S.Manoharan, M.Valan Rajkumar, VLSI Based Space Vector Pulse Width Modulation Switching Control in the proceedings of IEEE International Conference on Advanced Communication Control and Computing Technologies ICACCCT 2012 on August 2012, ISBN No. 978-1-4673-2045-0 (Print) (Page):366-370.

[11] M.Valan Rajkumar, P.S.Manoharan, Space Vector Pulse Width Modulation of Three-Phase DCMLI with Neuro-Fuzzy MPPT for Photovoltaic System, World Journal of Modelling and Simulation, Volume 10, No 3, August 2014, Pages 193-205.

[12] Tan C.S.,Baharuddin I. "Study of Fuzzy And PI Controller for permanent magnet brushless de motor drive". Proceedings of international power Engineering and Optimization Conference, June, 23-24,2010,pp.517-521.

[13] M.Valan Rajkumar, Prakasam, P. and Manoharan, P.S. (2016) Investigational Validation of PV Based DCDMLI Using Simplified SVM Algorithm Utilizing FPGA Tied with Independent Sources. Circuits and Systems, Volume 7, No 11, 3831-3848. http://dx.doi.org/10.4236/cs.2016.711320

[14] Bhat,A.K.S.,1994. Analysis and Design of LCL- Type Series Resonant Converter ,IEEE Trans Power Electronics,8:1-11.

[15] K.S.Tey and S. Mekhilef, "Modified incremental conductance MPPT algorithm to mitigate inaccurate responses under fast changing solar irradiation level," Solar Energy, vol. 101, pp. 333 to 342, Jan. 2014.

[16] M.Valan Rajkumar, P.S.Manoharan, "Modeling, Simulation and Harmonic Reduction of Three-Phase Multilevel Cascaded Inverters with SVPWM for Photovoltaic System”, Journal International Review on Modeling and Simulations, Volume 6, No. 2, April 2013, Pages 342-350. ISSN: 1974-9821 (Print), 1974-982X (Online)

[17] M.Valan Rajkumar, P.S.Manoharan, "Modeling and Simulation of Five-level Five-phase Voltage Source Inverter for Photovoltaic Systems", Journal Przeglad Elektrotechniczny, Volume 10, No. 10, October 2013, Pages 237-241. ISSN: 0033-2097 (Print)

[18] A.Ravi, P.S.Manoharan, M.Valan Rajkumar, "Harmonic Reduction of Three-Phase Multilevel Inverter for Grid connected Photovoltaic System using Closed Loop Switching Control”, Journal-IREMOS, Volume 5, No 5, October 2012, Pages 1934-1942. ISSN: 1974-9821 (Print), 1974-982X (Online)

[19] P.Thirumurugan, P.S.Manoharan, M.Valan Rajkumar, "VLSI Based Inverter Switching Control" in the proceedings of International Conference on Mathematical Modeling and Applied Soft Computing MMASC'12 - Coimbatore Institute of Technology on July 2012, Vol-2 (Page):965-973.

[20] S. Mirjalili, S. M. Mirjalili, and A. Lewis, "Grey wolf optimizer," Adv.Eng. Software, vol. 69, pp. 46-61, 2014.

[21] C.Hemalatha, M.Valan Rajkumar, G.Vidhya Krishnan, "Simulation and Analysis for MPPT Control with Modified firefly algorithm for photovoltaic system”, International Journal of Innovative Studies in Sciences and Engineering Technology, Volume 2, No 11, Nov.2016, Pages 48-52.

[22] G.Vidhya Krishnan, M.Valan Rajkumar, C.Hemalatha, "Modeling and Simulation of 13-level Cascaded Hybrid Multilevel Inverter with less number of Switches", International Journal of Innovative Studies in Sciences and Engineering Technology, Volume 2, No 11, Nov.2016, Pages 43-47.

[23] B. Sanjay Gandhi, S. Sam Chelladurai, and Dr. D. Senthil Kumaran, "Process Optimization for Biodiesel Synthesis from Jatropha Curcas Oil”, Taylor \& Francis-Distributed Generation and Alternative Energy Journal, Vol.23, No.4, Page 6- 16, 2011.

[24] B. Sanjay Gandhi and D. Senthil Kumaran, "The Production and Optimization of Biodiesel from Crude Jatropha Curcas Oil by a Two Step Process - An Indian Case Study Using Response Surface Methodology”, Taylor \& Francis-International Journal of Green Energy, Vol.113, No.10, Page 1084-1096, 2014.

[25] S. Chandrasekar and Gian Carlo Montanari, "Analysis of Partial Discharge Characteristics of Natural Esters as Dielectric Fluid for Electric Power Apparatus Applications,” IEEE Transactions on Dielectrics and Electrical Insulation, Vol. 21, No. 3, pp.1251-1259, June 2014.

[26] V.Jayaprakash Narayanan, B.Karthik and S.Chandrasekar," Flashover Prediction of Polymeric Insulators Using PD Signal TimeFrequency Analysis and BPA Neural Network Technique," Journal of Electrical Engineering and Technology. Vol. 9, Issue 4, pp. 1375-1384, 2014 